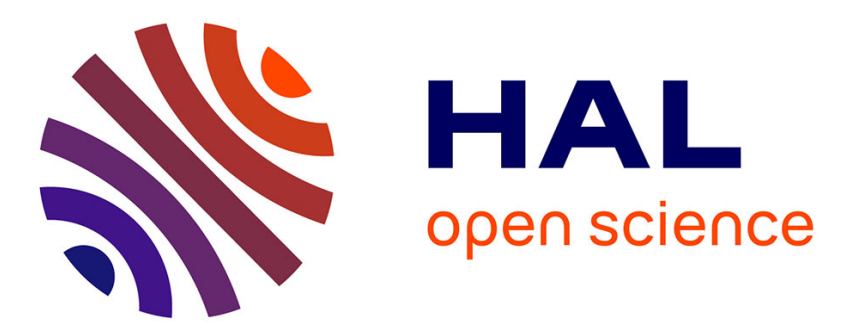

\title{
Blood cell - vessel wall interactions probed by reflection interference contrast microscopy
}

Heather Davies, Natalia Baranova, Nouha El Amri, Liliane Coche-Guerente, Claude Verdier, Lionel Bureau, Ralf Richter, Delphine Débarre

\section{- To cite this version:}

Heather Davies, Natalia Baranova, Nouha El Amri, Liliane Coche-Guerente, Claude Verdier, et al.. Blood cell - vessel wall interactions probed by reflection interference contrast microscopy. EUROPEAN CONFERENCES ON BIOMEDICAL OPTICS :Advances in Microscopic Imaging, Jun 2019, Munich, France. 10.1117/12.2527058 . hal-02368135

\section{HAL Id: hal-02368135 \\ https://hal.science/hal-02368135}

Submitted on 26 Nov 2019

HAL is a multi-disciplinary open access archive for the deposit and dissemination of scientific research documents, whether they are published or not. The documents may come from teaching and research institutions in France or abroad, or from public or private research centers.
L'archive ouverte pluridisciplinaire $\mathbf{H A L}$, est destinée au dépôt et à la diffusion de documents scientifiques de niveau recherche, publiés ou non, émanant des établissements d'enseignement et de recherche français ou étrangers, des laboratoires publics ou privés. 


\section{Blood cell - vessel wall interactions probed by reflection interference contrast microscopy}

Heather S. Davies, Natalia S. Baranova, Nouha El Amri, Liliane Coche-Guérente, Claude Verdier, et al.

Heather S. Davies, Natalia S. Baranova, Nouha El Amri, Liliane CocheGuérente, Claude Verdier, Lionel Bureau, Ralf P. Richter, Delphine Débarre, "Blood cell - vessel wall interactions probed by reflection interference contrast microscopy," Proc. SPIE 11076, Advances in Microscopic Imaging II, 110760V (22 July 2019); doi: 10.1117/12.2527058 


\title{
Blood cell - vessel wall interactions probed by Reflection Interference Contrast Microscopy
}

\author{
Heather S. Davies ${ }^{1}$, Natalia S. Baranova ${ }^{2,3}$, Nouha El Amri ${ }^{1}$, Liliane Coche-Guerente ${ }^{4}$, Claude \\ Verdier $^{1}$, Lionel Bureau ${ }^{1}$, Ralf P. Richter ${ }^{2,5}$, and Delphine Débarre ${ }^{1, *}$ \\ ${ }^{1}$ Université Grenoble Alpes, CNRS, Laboratoire Interdisciplinaire de Physique (LIPhy), 38000 \\ Grenoble, France; ${ }^{2}$ Biosurface Lab, CIC biomaGUNE, Paseo Miramon 182, 20014 San Sebastian, \\ Spain; ${ }^{3}$ Current address: Institute of Science and Technology Austria (IST Austria), Am Campus 1, \\ 3400 Klosterneuburg, Austria; ${ }^{4}$ Université Grenoble Alpes, CNRS, Département de Chimie \\ Moléculaire (DCM), Grenoble, France; ${ }^{5}$ School of Biomedical Sciences, Faculty of Biological \\ Sciences, School of Physics and Astronomy, Faculty of Mathematics and Physical Sciences, Astbury \\ Centre for Structural Molecular Biology, University of Leeds, Leeds LS2 9JT, United Kingdom. \\ *delphine.debarre@univ-grenoble-alpes.fr
}

\begin{abstract}
Numerous biophysical questions require the quantification of short-range interactions between (functionalized) surfaces and synthetic or biological objects such as cells. Here, we present an original, custom built setup for reflection interference contrast microscopy that can assess distances between a substrate and a flowing object at high speed with nanometric accuracy. We demonstrate its use to decipher the complex biochemical and mechanical interplay regulating blood cell homing at the vessel wall in the microcirculation using an in vitro approach. We show that in the absence of specific biochemical interactions, flowing cells are repelled from the soft layer lining the vessel wall, contributing to red blood cell repulsion in vivo. In contrast, this so-called glycocalyx stabilizes rolling of cells under flow in the presence of a specific receptor naturally present on activated leucocytes and a number of cancer cell lines.
\end{abstract}

Keywords: Reflection interference contrast microscopy; high speed tracking; cell-surface interactions.

\section{REFLECTION INTERFERENCE CONTRAST MICROSCOPY}

Monitoring interactions between objects and a nearby surface is a key aspect of soft matter studies ranging from wetting to complex fluid flow. In biophysics, these interactions govern cell adhesion or the flow of microswimmers or blood cells. The non invasive investigation of such short-range interactions in nevertheless challenging, in particular under flow where fast imaging is required: while TIRF has been used extensively for cell adhesion studies, it requires staining of the sample and the quantitative measurement of distances remains challenging. Here, we demonstrate the use of Reflection Interference Contrast Microscopy (RICM) to probe interactions between blood cells (or blood cell mimetics) and functionalized surfaces displaying mechanical and biochemical properties similar to the blood vessel walls.

RICM is an optical technique that can probe optical distances between two or more interfaces based on the interference pattern formed by the light reflected from these interfaces. In its simplest form, it consists in illuminating the sample with nearly parallel, partially coherent light that is reflected by the substrate (e.g. the upper face of a glass coverslip) and the object under investigation: a cell, bead or vesicle adhering or hovering over this substrate [1]. The intensity pattern is imaged on a camera and analyzed in terms of difference in optical path lengths between the two reflections that give rise to constructive or destructive interferences (Fig. 1). RICM imaging can provide information on the height profile of complex samples such as vesicles and cells. In this case, the shape of the object under investigation is unknown, and the intensity profile is used to determine the height of the object at different positions by use of a priori knowledge of the object optical properties or previous calibration (Fig. 5d). This approach has been successfully used for studying adhesion of unilamellar vesicles and cells, or protein or electrolyte layer deposition on surfaces [1,2]. Alternatively, RICM can be used to automatically track objects of well-defined shape and optical properties such as beads with an accuracy of $\sim 5 \mathrm{~nm}$ in all directions.

Here, in order to investigate the mechanical and biochemical aspects of blood cell - vessel wall interactions, we have designed and built an original RIC microscope allowing multimodal imaging of our sample at high frame rate. Coupled 
to a careful analysis of the data, this has allowed us to demonstrate experimentally the role of the softness of the vessel wall outer layer in the repulsion of non-interacting cells.

Our custom-built experimental setup is shown on Fig. 1. In contrast to previous implementations of RICM, it decouples polarisation optics from the objective, allowing complete freedom on the choice of magnification, immersion medium and chromatic aberration correction. Coupled to a broadband, continuous white light source this has allowed us to build a flexible setup allowing multicolour RICM images or its straightforward combination with other modalities such as brightfield, phase contrast or fluorescence imaging. We demonstrate its use on the precision tracking of functionalized microspheres at high speed that can be used as cell mimetics for in vitro studies.

(a)

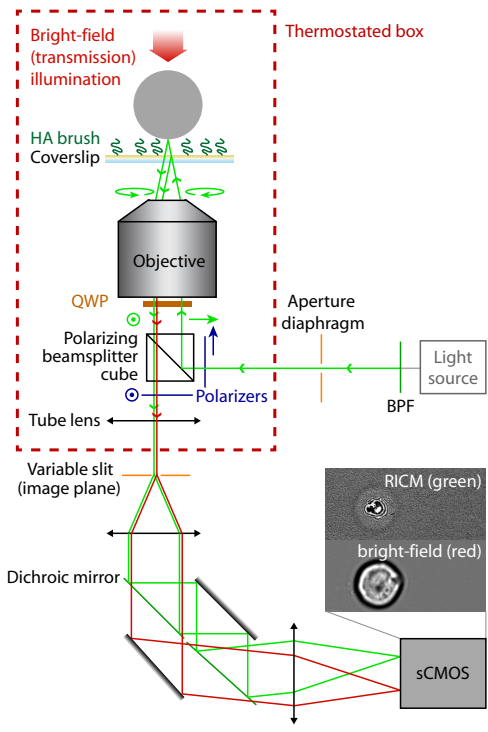

(b)

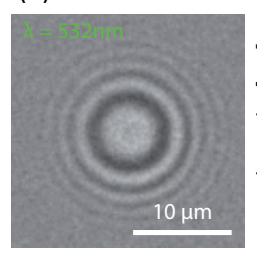

(c)

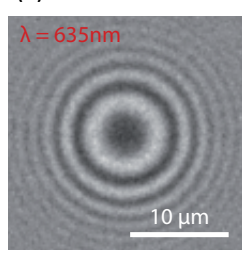

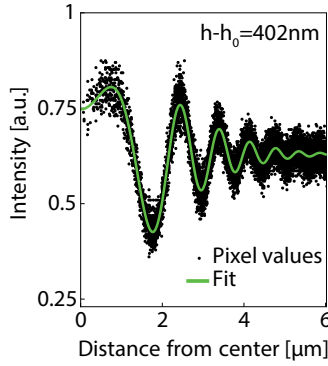

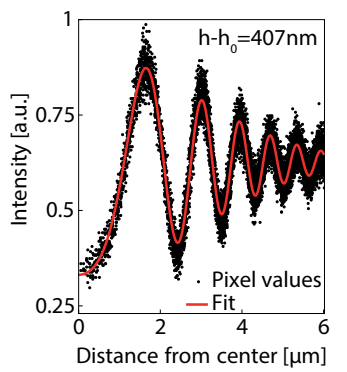

(d) $\mathrm{h}[\mathrm{nm}]$

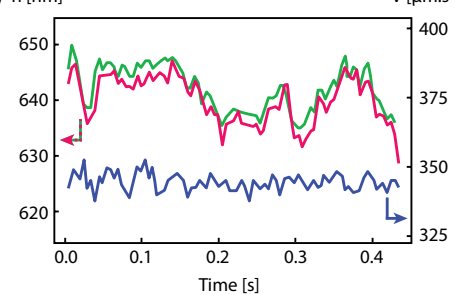

(e)

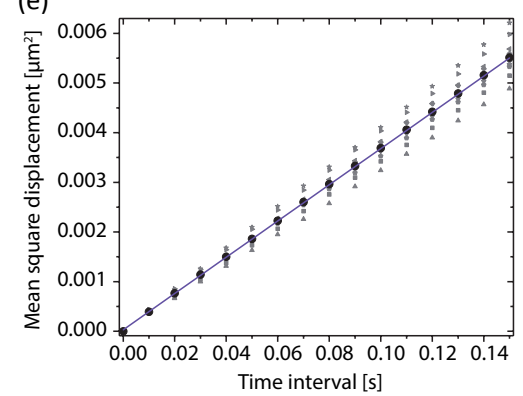

Figure 1. Multimodal RICM setup and bead tracking. (a), schematic view of the multimodal microscope. BPF, dichroic bandpass filters. QWP, imaging-flat quarter waveplate. For two- or three- color imaging at high speed, a home-built image-splitting setup is used to simultaneously acquire spectrally separated images on a sCMOS camera. Here, an example of combined RICM (green)/ brightfield (red) imaging is shown. (b-c), typical RICM images of $15-\mu \mathrm{m}$ polystyrene beads at two diffent wavelengths. Azimuthal averages of the curves can be fitted to retrieve the bead-substrate distance with an accuracy of a few $\mathrm{nm}$ after localization of the pattern center that permits in-plane tracking of the bead. (d), example of the resulting height trajectories (green, $532 \mathrm{~nm}$ and red, $635 \mathrm{~nm}$ ) and speed (blue) under flow. (e), analysing the mean square displacement of Brownian beads without flows permits quantifying the precision of in-plane localization as the offset of the curve (here $\sim 5 \mathrm{~nm} \mathrm{rms}$ ). Adapted from [3].

\section{IN VITRO PROBING OF CELL-VESSEL WALL INTERACTIONS UNDER FLOW}

Next, we discuss the application of this method to the study of blood cell - vessel wall interactions under flow. This interaction is highly regulated as it is both critical in the flow of red blood cells and in the control of white blood cell adhesion to the walls e.g. at a site of inflammation. However, the biochemical and mechanical cues governing this regulation are still poorly understood. In particular, endothelial cells lining the blood vessels are covered with a thick and highly deformable polymer layer called glycocalyx that is first encountered by flowing cells when interacting with the vessel wall. To decipher the essential parameters controlling cell homing or repulsion, we have developed an in vitro platform allowing precise control of the surface functionalization (by a glycopolymer brush) mimicking the physiological glycocalyx and of the flow of cell mimetics that can display specific receptors of glycocalyx components, while permiting direct imaging of these mimetics at high speed [3].

Based on this platform, we have studied how the interplay between mechanical properties of the brush and specific biochemical interactions regulate cell-surface interactions under flow. First, we have shown that in the absence of specific biochemical interactions, the microspheres mimicking blood cells are repelled from the brush when submitted to a strong enough shear flow. The equilibrium distance can reach up to several hundreds of nanometers under physiological shear stresses, significantly contributing to limiting cell adhesion and to the margination of red blood cells 
experimentally observed in vivo in microcirculation. These results are well explained by the theory of elastohydrodynamics that accounts for the effect of substrate deformation under hydrodynamic forces, providing the first experimental evidence of this "soft biolubrication" effect at play at small scale [4].

We then show how the introduction of specific binding to the hyaluronan brush serving as glycocalyx model through functionalization of the microspheres with CD44 receptors stabilizes bead rolling under physiologically-relevant flow fields. This rolling is strongly dependent on physical parameters of the brush (thickness and softness), providing insight into the mechanisms of control of cell homing by the glycocalyx in the initial stages of the process.

Finally, we show how these results can be extended to probing the interaction of real white blood cells with our model surface, confirming the mechanisms unveiled with our fully synthetic model.

(a)

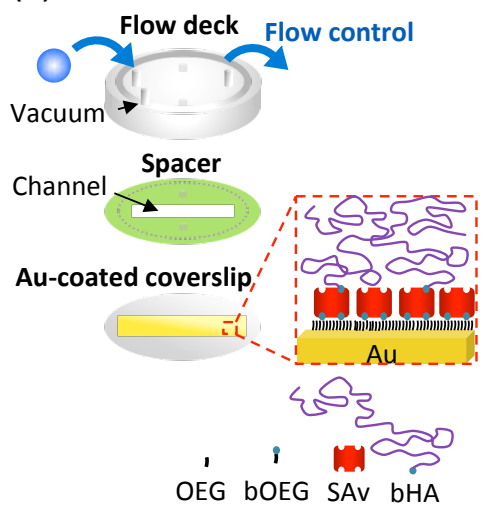

(b) shear flow

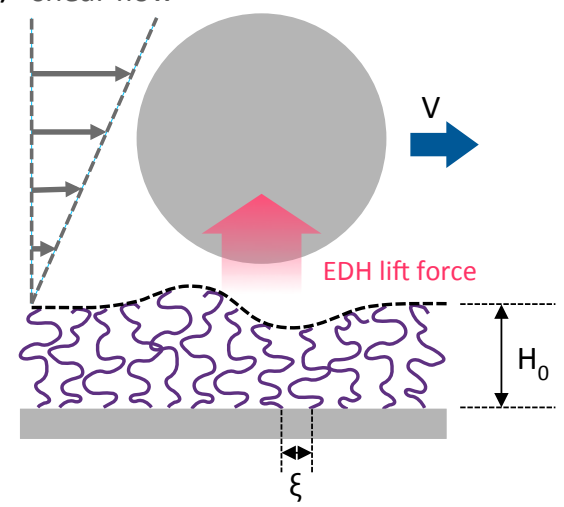

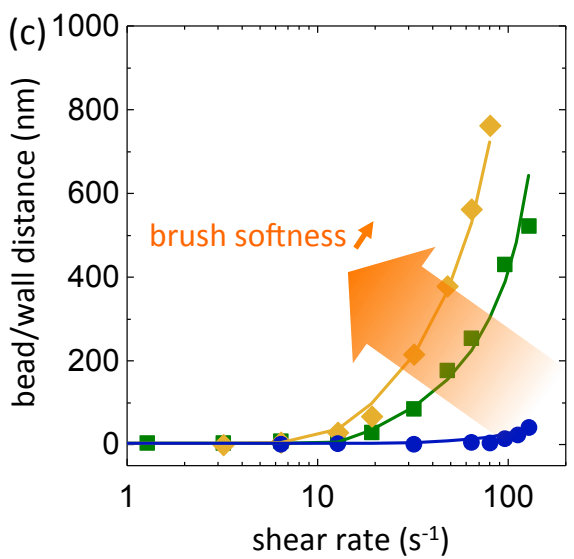

Figure 2. (a), in vitro platform for the study of glycocalyx model - cell mimetics interactions. A flow chamber coupled to a syringe pump is used for in situ surface functionalization and subsequent flow control. $15-\mu \mathrm{m}$ microspheres are used as cell mimetics after functionalization with a well controlled surface density of CD44 receptors. (b)OEG, (biotinylated) oligo ethylene glycol; SAv, streptavidin; bHA, end-biotinylated hyaluronic acid of controlled molecular weight. Optical observation is possible through the bottom coverslip and the transparent flow deck. Adapted from [3]. (b), schematic representation of the bead-model layer interaction under a shear flow in the absence of CD44 functionalization. The magnitude of the elastohydrodynamic (EDH) lift depends on the shear stress but also on the brush parameters: thickness, grafting density. (c), experimental (symbols) and theoretical (plain line) EDH-induced equilibrium bead/wall distance vs. shear rate for different HA brushes of varying elastic properties (blue, $15 \mathrm{kPa}$; green, $40 \mathrm{~Pa}$; Yellow, $8 \mathrm{~Pa}$ ): softer brushes induce a lift of greater magnitude. Adapted from [4].

\section{REFERENCES}

[1] Limozin, L. and Sengupta, K., "Quantitative reflection interference contrast Microscopy (RICM) in soft matter and cell adhesion," ChemPhysChem 10, 2752-2768 (2009).

[2] Valignat, M.-P., "Biomolecular Interaction Studies using SEEC Technology", Biophys. J., 114, 186a-187a (2018).

[3] Davies, H. D, Baranova, N. S., El Amri, N., Coche-Guérente, L., Verdier, C., Bureau, L., Richter, R. P., and Débarre, D., "An integrated assay to probe endothelial glycocalyx-blood cell interactions under flow in mechanically and biochemically well-defined environments," to be published in Matrix Biology (2019).

[4] Davies, H. D., Débarre, D., El Amri, N., Verdier, C., Richter, R.P., and Bureau, L., "Elastohydrodynamic lift at a soft wall," Physical Review Letters 120, 198001 (2018). 